\title{
Comparison between pitch discrimination in normal children, children with hearing aids, and children with cochlear implant
}

Rania E. Ahmed

\author{
AudioVestibular Unit, Department of \\ Otorhinolaryngology, Alexandria Univesity, \\ Alexandria, Egypt \\ Correspondence to Rania E. Ahmed, MSC, 11 \\ ELMohasba, st, Bolekly, Alexandria. Tel: \\ 01224030276; \\ e-mail: raniaebraheem1985@gmail.com \\ Received 17 September 2018 \\ Accepted 20 August 2018 \\ The Egyptian Journal of Otolaryngology \\ 2018, 34:332-336
}

\author{
Background \\ Materials and Methods \\ Results \\ language development than HA. \\ Conclusions \\ outweighs the deficit in pitch discrimination. \\ Keywords: \\ pitch, hearing aids, cochlear implant, language \\ Egypt J Otolaryngol 34:332-336 \\ (C) 2018 The Egyptian Journal of Otolaryngology \\ $1012-5574$
}

Cochlear hearing loss causes variations in the way that sounds are represented in the auditory system and for cochlear implant $(\mathrm{Cl})$ users, pitch information that is transmitted to the central nervous system is not ideal. The aim of this study was to compare between pitch discrimination and its associated language development in normal children, children with cochlear implant and children with hearing aids to know which prostheses is more useful to the patient.

The study measured pitch discrimination test, just noticeable difference test and language evaluation in 45 children divided into 3 groups.

This study suggested that $\mathrm{Cl}$ had less pitch discrimination ability but better

The benefit that $\mathrm{Cl}$ users get through better accessibility to high frequencies

\section{Introduction}

Pitch is a main attribute of any auditory stimulus. It is controversially the most related perceptual measurement in most types of western music and is also of great importance for speech communication, carrying important prosody information in languages such as English and also conveying semantic details in tone languages, such as Mandarin.

The cochlea carries out a spectral analysis of the incoming sound and maps stimulus frequency onto place along the basilar membrane. These mapped frequencies are then signaled to the brain via the auditory nerve.

Speech perception is achieved by the extraction of independent characteristics which are then merged over time by a synthesis operation into a phonetic or syllabic percept. It is a dynamic operation, and it has been shown that parts of the auditory system are capable of doing physiological alternations that coincide with perceptual changes. Normal speech perception is based on intact encoding of acoustic change [1].

However, for cochlear implant (CI) users pitch information that is transferred to the central nervous system is not ideal. CI users therefore have considerable trouble recognizing pitch differences than their normal-hearing counterparts do [2].
The poor pitch discrimination is an important cause of the great problems in music appreciation, understanding of speech in noise, and in understanding of tonal language. One evident reason for poor pitch discrimination in CI users may be the lack of sharp frequency tuning in electric hearing [3]. sensory Neural Hearing loss (SNHL) listeners always face a problem in their ability to discriminate the f0 of complex sounds [3].

Users of modern CI generally have good performance in quiet listening conditions with good users being able to communicate on the telephone. However, these individuals still have poor performance in speech recognition in noise, music appreciation, and in understanding of tonal language.

Accordingly, the aim of this study was to compare pitch discrimination and its associated language development in normal children, children with CIs and children with hearing aids (HA) to know which prostheses is more useful to the hearing loss patient in their ability to perceive and discriminate pitch.

This is an open access journal, and articles are distributed under the terms of the Creative Commons Attribution-NonCommercial-ShareAlike 4.0 License, which allows others to remix, tweak, and build upon the work non-commercially, as long as appropriate credit is given and the new creations are licensed under the identical terms. 


\section{Patients and methods}

\section{Patients}

This study was carried out in the Audiology Unit of Alexandria Main University Hospital on 45 children aged from 10 to 18 years who were divided into a group of children with HA, a group of children with CIs, and a group of children with normal hearing as a control group.

The HA group which consisted of 15 patients were wearing the HA since at least 1 year. The CI group consisted of 15 patients who were implanted since at least 1 year and wear implants before 6 years. The children in both groups were receiving regular speech therapy for a minimum of 1 year. The control group consisted of 15 patients with normal hearing. The patients of the three groups had average IQ score.

The patients of the two hearing loss groups attended the Audiology Unit at Alexandria Main University Hospital for follow up of their devices and/or programming and they had severe to profound SNHL.

\section{Methods}

Medical ethics were considered and the patients were informed that they will be a part of a research study and were asked to sign written consent and the study took the IRB approval from Alexandria University.

(1) All patients were subjected to history taking, otoscopic examination, assessment of peripheral hearing sensitivity by measuring $\mathrm{AC}$ thresholds for frequency range $250-8000 \mathrm{~Hz}$, bone conduction for frequency range $250-4000 \mathrm{~Hz}$, and aided freefield air conduction thresholds for the $\mathrm{HA}$ and $\mathrm{CI}$ groups.

(2) Stanford-Binet psychometric test (only nonverbal testing) and language assessment tests (Comprehensive Arabic Language Test).

(3) Pitch discrimination test (PDT): the test contained 20 pairs of tone bursts and intertone intervals. The tones in each pair were combinations of two frequencies, 880 and $1122 \mathrm{~Hz}$, which are designated a low frequency (L) and a high frequency $(\mathrm{H})$, respectively. Thus, there are four possible combinations (LH, LL, $\mathrm{HL}$, and $\mathrm{HH})$. The pairs were presented with $\sim 6$-s interpair intervals. The patient was instructed that they will hear sets of two consecutive tones that varied in pitch. The task of the patient was to discriminate the pattern of the two tones (LL, HL, HH, LH) and if the patient discriminate inverted pattern it was considered satisfactory. The correct score was calculated in a percentage and was recorded in a uniform scoring sheet. The test duration was about 5-8 min.

(4) Just noticeable difference (JND) frequency test: the test was able to find out the minimum change in frequency needed by the patient to hear the difference between frequencies (JND). Ranging from 500 to $4000 \mathrm{~Hz}$, taking $500 \mathrm{~Hz}$ as low frequency, $2000 \mathrm{~Hz}$ as medium frequency, and $4000 \mathrm{~Hz}$ as high frequency. The patients were instructed that they will hear sets of two consecutive tones that varied in pitch, play each frequency with sets of increment $1 \%$ as $1-50 \%$, present the baseline frequency and (baseline frequency and increment) in pairs with each frequency being $0.5 \mathrm{~s}$ with pause in between $0.3 \mathrm{~s}$ and pause between each pair being $5 \mathrm{~s}$. The task of the patient was to judge if and when he will hear a change in pitch between the pair. The correct score was considered as a threshold (the level at which the patient just can notice a difference between the two tones and cannot notice it before and can notice it in each pair after it).

\section{Results and discussion}

The aim of this study was to compare the pitch discrimination and associated language development relative to it in normal children. It is well known that children with CIs perform better than those using HA.

This study was a prospective study. It was conducted on 45 patients ( 23 girls and 22 boys) who were divided into three groups of 15 children each. The first group was the HA children, the second group CIs children, and the third is the control normal-hearing children. Age of the patients ranged from 10 to 18 years. All patients included in this study had average performance (abstract) and general IQ (this was one of the selection criteria of the study).

\section{Language test}

Table 1 shows that the total scores of the language test (Comprehensive Arabic Language Test) were

Table 1 Comparison between the two groups according to language test

\begin{tabular}{lcccc}
\hline & $\mathrm{Cl}(n=15)$ & $\mathrm{HA}(n=15)$ & $U$ & $P$ \\
\hline Language test & & & & \\
Minimum- & $257.0-359.0$ & $149.0-350.0$ & $55.50^{*}$ & $0.018^{*}$ \\
maximum & & & & \\
Mean \pm SD & $339.4 \pm 27.6$ & $302.2 \pm 67.70$ & & \\
Median & 348.0 & 338.0 & & \\
\hline
\end{tabular}

$\mathrm{Cl}$, cochlear implant; $\mathrm{HA}$, hearing aids; $U, P, U$ and $P$ values for Mann-Whitney test for comparing between the two groups.

"Statistically significant at $P$ value less than or equal to 0.05 . 
significantly higher in CI group than in the HA group. Therefore, speech development in the CI group was better than the speech development in the HA group and this can be explained by knowing that slope SNHL is the most common type to be found in clinical audiology practice; the cases included in the study had the slope SNHL configuration. The frequencies 500, 1000, and $2000 \mathrm{~Hz}$ are believed to be closely connected to speech. Vowels and consonants, the elementary units of speech, have different spectral characteristics. Vowels are naturally more intense and carry acoustic energy at low frequencies $(400-500 \mathrm{~Hz})$ [4]. Consonants, on the other hand, are sounds having spectral energy at high frequencies, above $2000 \mathrm{~Hz}$ [5]. However, speech intelligibility is based on consonant sounds which contribute $60 \%$ of it, while vowels contribute only $40 \%$ [6]. So we can understand that the cause of impaired speech recognition in individuals with highfrequency hearing loss is the spectral characteristics of these sounds and the range of human audibility [7].

The acoustic stimulation in the HA group just amplifies the frequencies and so the aided audiogram will still have a drop in the high frequencies than the low frequencies while the electrical stimulation in the CI group damage all the residual hearing and stimulate all frequencies like each other, so the high frequencies here will have a significant increase and this is the cause of the results we had. Our result was in agreement with those of several studies reported that the speech recognition has the highest correlation with the high frequencies as approved by Anjos et al. [7].

\section{Pitch discrimination}

(1) PDT.

(2) JND test.

Both the tests reflect spectral discrimination abilities for eliciting stimuli, we used the JND test to ensure the results as this test compares the pitch discrimination ability by using very close frequencies so it makes pitch discrimination more difficult.

As shown in Tables 2 and 3 the scores of these tests were significantly decremented in both subgroups of the study than the control group and this can be

Table 2 Comparison between the three groups according to pitch discrimination test

\begin{tabular}{|c|c|c|c|c|c|}
\hline & Normal $(n=15)$ & $\mathrm{Cl}(n=15)$ & $\mathrm{HA}(n=15)$ & $F$ & $P$ \\
\hline \multicolumn{6}{|l|}{ PDT (\%) } \\
\hline Minimum-maximum & $90.0-100.0$ & $70.0-85.0$ & $70.0-100.0$ & $41.772^{*}$ & $<0.001^{*}$ \\
\hline Mean \pm SD & $96.33 \pm 4.42$ & $75.33 \pm 4.81$ & $82.0 \pm 9.02$ & & \\
\hline Median & 100.0 & 75.0 & 80.0 & & \\
\hline Significance between groups & \multicolumn{4}{|c|}{$P_{1}<0.001^{*}, P_{2}<0.001^{*}, P_{3}<0.001^{*}$} & \\
\hline
\end{tabular}

$\mathrm{Cl}$, cochlear implant; $F, F$ value for analysis of variance test, significance between groups was done using the post-hoc test (least significance difference); HA, hearing aids; PDT, pitch discrimination test. $P_{1}, P$ value for comparing between normal and $\mathrm{Cl}$. $P_{2}, P$ value for comparing between normal and $\mathrm{HA} . P_{3}, P$ value for comparing between $\mathrm{Cl}$ and $\mathrm{HA}$. ${ }^{*}$ Statistically significant at $P$ value less than or equal to 0.05 .

Table 3 Comparison between the three groups according to just noticeable difference

\begin{tabular}{|c|c|c|c|c|c|}
\hline JND & Normal $(n=15)$ & $\mathrm{Cl}(n=15)$ & $\mathrm{HA}(n=15)$ & $\mathrm{KW}_{\chi^{2}}^{2}$ & $P$ \\
\hline \multicolumn{6}{|l|}{500} \\
\hline Minimum-maximum & $5.0-8.0$ & $20.0-45.0$ & 15.0-20.0 & $39.316^{*}$ & $<0.001^{*}$ \\
\hline Mean \pm SD & $6.20 \pm 1.01$ & $35.93 \pm 6.94$ & $16.60 \pm 1.50$ & & \\
\hline Median & 6.0 & 35.0 & 17.0 & & \\
\hline Significance between groups & \multicolumn{3}{|c|}{$P_{1}<0.001^{*}, P_{2}<0.001^{*}, P_{3}<0.001^{*}$} & & \\
\hline \multicolumn{6}{|l|}{2000} \\
\hline Minimum-maximum & 12.0-20.0 & $46.0-84.0$ & $24.0-32.0$ & $39.695^{\star}$ & $<0.001^{*}$ \\
\hline Mean \pm SD & $13.87 \pm 2.56$ & $71.33 \pm 10.24$ & $27.73 \pm 3.53$ & & \\
\hline Median & 12.0 & 72.0 & 28.0 & & \\
\hline Significance between groups & \multicolumn{3}{|c|}{$P_{1}<0.001^{*}, P_{2}<0.001^{*}, P_{3}<0.001^{*}$} & & \\
\hline \multicolumn{6}{|l|}{4000} \\
\hline Minimum-maximum & $24.0-40.0$ & $88.0-112.0$ & $48.0-72.0$ & $39.618^{*}$ & $<0.001^{*}$ \\
\hline Mean \pm SD & $29.33 \pm 5.79$ & $98.67 \pm 8.90$ & $60.27 \pm 7.32$ & & \\
\hline Median & 32.0 & 96.0 & 64.0 & & \\
\hline Significance between groups & \multicolumn{3}{|c|}{$P_{1}<0.001^{*}, P_{2}<0.001^{*}, P_{3}<0.001^{*}$} & & \\
\hline
\end{tabular}

$\mathrm{Cl}$, cochlear implant; HA, hearing aids; JND, Just noticeable difference; KW, Kruskal-Wallis test, Significance between groups was done using Mann-Whitney test. $P_{1}, P$ value for comparing between normal and $\mathrm{Cl} . P_{2}, P$ value for comparing between normal and $\mathrm{HA}$. $P_{3}, P$ value for comparing between $\mathrm{Cl}$ and $\mathrm{HA}$. ${ }^{*}$ Statistically significant at $P$ value less than or equal to 0.05 . 
discussed by knowing that hearing impairment is frequently accompanied by poorer frequency selectivity, in the form of broader auditory filters. According to the place theory of pitch, poorer frequency selectivity should lead to poorer pitch perception $[8,9]$.

The results have also shown that the scores were significantly incremented in the HA group than in the CI group. CIs with multiple electrode stimulation can produce good speech perception but poor pitch perception, the poorer pitch discrimination results for CI patients compared with HA users and control groups can be attributed to the limited numbers of electrodes (12 in Medel/16 in Advanced Bionic) which would result in poorer frequency selectivity that leads to poorer pitch discrimination ability.

On the other hand, many individuals with severe or profound hearing loss have residual hearing in the lower frequency region, and this residual hearing was the cause of the increment of PDT and JND results in the HA group than the CI group; the residual hearing is responsible for the pitch discrimination ability. On the other hand, this residual hearing is always damaged by cochlear implantation and so the CI with multiple electrode stimulation deprives its patient from these residual hearing; decreasing his ability for pitch discrimination. This result is in agreement with those of several studies such as Oxenham [10], who correlated between the pitch perception ability and the low-frequency residual hearing accuracy.

As shown in Table 4, JND scores and aided thresholds of frequency $500 \mathrm{~Hz}$ were positively correlated in the HA group only and not correlated in the $\mathrm{CI}$ group or in the other frequencies in the HA group and this underlines that the HA group's better pitch discrimination abilities are strongly related to the residual hearing in low frequencies and the worse discrimination ability of the CI group was due to the poorer frequency selectivity and so they were not correlated to the aided thresholds [10].

As shown in Table 5, the results of correlation showed that the JND scores and language test scores were not correlated in the two groups and this can be explained by understanding that despite pitch and language processing are processed centrally together in the temporal lobe but pitch discrimination process is done totally peripheral in the cochlea depended on the psychoacoustics of place theory and temporal theory, most recent theories consider that the
Table 4 Correlation between just noticeable difference and aided thresholds

\begin{tabular}{lcccc}
\hline & \multicolumn{4}{c}{ Thresholds of aided vs. JND } \\
\cline { 2 - 5 } & \multicolumn{3}{c}{$\mathrm{Cl}$} & \multicolumn{3}{c}{$\mathrm{HA}$} \\
\hline JND & $r_{\mathrm{s}}$ & $P$ & $r_{\mathrm{s}}$ & $P$ \\
500 & 0.094 & 0.740 & $0.657^{\star}$ & $0.008^{\star}$ \\
2000 & 0.049 & 0.863 & 0.249 & 0.370 \\
4000 & -0.045 & 0.874 & $0.846^{*}$ & $<0.001^{*}$ \\
\hline
\end{tabular}

$\mathrm{Cl}$, cochlear implant; $\mathrm{HA}$, hearing aids; JND, Just noticeable difference; $r_{\mathrm{s}}$, Spearman's coefficient. *Statistically significant at $P$ value less than or equal to 0.05 .

Table 5 Correlation between just noticeable difference and language test

\begin{tabular}{lcccc}
\hline & \multicolumn{5}{c}{ Language } \\
\cline { 2 - 5 } & \multicolumn{3}{c}{$\mathrm{Cl}$} & \multicolumn{3}{c}{$\mathrm{HA}$} \\
\hline JND & $r_{\mathrm{s}}$ & $P$ & $r_{\mathrm{s}}$ & $P$ \\
500 & 0.23 & 0.860 & -10.4 & 0.702 \\
2000 & -0.442 & 0.530 & 1.19 & 0.893 \\
4000 & 0.638 & 0.473 & -8.95 & 0.173 \\
\hline
\end{tabular}

$\mathrm{Cl}$, cochlear implant; $\mathrm{HA}$, hearing aids; just noticeable difference; $r_{\mathrm{s}}$, Spearman's coefficient. * $S$ tatistically significant at $P$ value less than or equal to 0.05 .

Table 6 Correlation between the aided age and the language score in hearing aids and cochlear implant groups

\begin{tabular}{lcc}
\hline & $\mathrm{HA}(n=15)$ & $\mathrm{Cl}(n=15)$ \\
\hline Aided age & & \\
Minimum-maximum & $1.0-5.0$ & $3.0-7.0$ \\
Mean \pm SD & $3.43 \pm 1.02$ & $4.4 \pm 1.14$ \\
Median & 4.0 & 4.0 \\
Language score & & \\
$\quad$ Minimum-maximum & $149-350$ & $257-359$ \\
Mean \pm SD & $302.2 \pm 67.7$ & $339.4 \pm 27.6$ \\
Median & 338 & 348 \\
$r_{\mathrm{s}}$ & $-35.7^{*}$ & $-21.38^{*}$ \\
$P$ & $0.04^{*}$ & $<0.001^{*}$ \\
\hline
\end{tabular}

$\mathrm{Cl}$, cochlear implant; $\mathrm{HA}$, hearing aids; $r_{\mathrm{s}}$, Spearman's coefficient. *Statistically significant at $P$ value less than or equal to 0.05 .

process of language is carried out entirely inside the brain. Studies have shown that most of the language processing functions are carried out directly in the central temporal lobe of the cerebral cortex $[11,12]$. As shown in Table 6, the results of correlation showed that the aided age and the language score were negatively correlated in the two groups and this can be explained by understanding that the earlier the age the patient had been aided in, the better the language development that occurred [13].

\section{Conclusions}

Our study supported that the benefit that CI users get through better accessibility to high frequencies outweighs the deficit in pitch discrimination. 


\section{Financial support and sponsorship}

Nil.

\section{Conflicts of interest}

There is no conflict of interest.

\section{References}

1 Carroll J, Zeng FG. Fundamental frequency discrimination and speech perception in noise in cochlear implant simulations. Hear Res 2007; 231:42-53.

2 Chen $\mathrm{H}$, Ishihara YC, Zeng FG. Pitch discrimination of patterned electric stimulation. J Acoust Soc Am 2005; 118:338-345.

3 McKinnon A. Pitch perception of young cochlear implant users and normal-hearing peers[unpublishedM. Sc.]. Canada:DalhousieUniversity;2007.

4 Russo ICP, Behlau M. Percepção da fala: análise acústica do português brasileiro, Lovise, São Paulo, 1993.
5 Staab W. Significance of mid-frequencies in hearing aid selection. Hear J 1988; 42:23-25.

6 Fletcher $\mathrm{H}$. Speech and hearing communication. New Jersey: D. Van Nostrand; 1953.

7 Anjos WTd, Ludimila L, Resende LMd, Costa-Guarisco LP. Correlation between the hearing loss classifications and speech recognition. Rev CEFAC 2014; 16:1109-1116.

8 Glasberg BR, Moore BC. Derivation of auditory filter shapes from notchednoise data. Hear Res 1990; 47:103-138.

9 Patterson RD. Auditory filter shapes derived with noise stimuli. J Acoust Soc Am 1976; 59:640-654.

10 Oxenham AJ. Pitch perception. J Neurosci 2012; 32:13335-13338.

11 Heeger D. Perception lecture notes: frequency tuning and pitch perception. New York: Department of Psychology, New York University; 2006.

12 Friederici $A D$. The brain basis of language processing: from structure to function. Physiol Rev 2011; 91:1357-1392.

13 Moeller MP. Early intervention and language development in children who are deaf and hard of hearing. Pediatrics 2000; 106:E43. 